\title{
Overview on the PHRESCO Project: PHotonic REServoir COmputing
}

\author{
Jean-Pierre Locquet ${ }^{1,2,3,4,5(\bowtie)}$ (1) and PHRESCO Partners ${ }^{1,2,3,4,5}$ \\ ${ }^{1}$ Katholieke Universiteit Leuven, Leuven, Belgium \\ jeanpierre. locquet@kuleuven. be \\ ${ }^{2}$ Universiteit Gent, Ghent, Belgium \\ 3 The Leibniz Institute for Innovative Microelectronics, Frankfurt, Germany \\ ${ }^{4}$ IBM Research GmbH, Rüschlikon, Switzerland \\ 5 The CentraleSupélec, Gif-sur-Yvette, France
}

\begin{abstract}
PHRESCO is an EU-H2020 funded project that was running for four years and will be ending in September 2019. PHRESCO focused on the development of efficient cognitive computing into a specific silicon-based technology by co-designing a new reservoir computing chip, including innovative electronic and photonic components that will enable major breakthrough in the field. So far, a first-generation reservoir with 18 nodes and integrated readout was designed, fabricated, characterized and a training method has been developed. Additionally, large efforts of the consortium were dedicated to the design of the second-generation chip consisting of larger networks (60 nodes), with an on-chip readout and novel training approaches. This short abstract provides key information on the status of the work achieved and discuss further the potential exploitation routes and the key barriers that still need to be removed to bring the technology to a higher maturity level. A part of the exit strategy of PHRESCO is to identify potential future cooperation with interested stakeholders who are willing to co-develop the PHRESCO technology together with the PHRESCO partners for bringing it to an exploitable or marketable system. This abstract lays down the foundations for potential exploitation activities with interested stakeholders.
\end{abstract}

Keywords: Reservoir computing $\cdot$ Cognitive computing $\cdot$ Machine learning

\section{Introduction}

New computing paradigms are required to feed the next revolution in Information and Communication Technology (ICT). Machines that can learn, but also handle vast amount of data, need to be invented. In order to achieve this goal and still reduce the energy footprint of ICT, fundamental hardware innovations must be done. A physical implementation natively supporting new computing methods is required. Most of the time, CMOS is used to emulate e.g. neuronal behavior, but is intrinsically limited in power efficiency and speed. Reservoir computing (RC) is one of the concepts [1] that

PHRESCO partners formed by Coordinator of the PHRESCO project. 
has proven its efficiency to perform tasks where traditional approaches fail. It is also one of the rare concepts of an efficient hardware realization of cognitive computing into a specific, silicon-based technology and of an efficient neural network paradigm suitable for time-series processing. Small RC systems have been demonstrated using optical fibers and bulk components. In 2014, optical RC networks based on integrated photonic circuits were demonstrated by one of the PHRESCO partners [2].

The PHRESCO project aims to bring photonic reservoir computing to the next level of maturity. A new RC chip was designed, including innovative electronic and photonic components that enabled major breakthrough in the field. The consortium of the project is composed by the Katholieke Universiteit Leuven (KUL) in Belgium, who is the coordinator, the Universiteit Gent (UGent) in Belgium, the Leibniz Institute for Innovative Microelectronics (IHP) in Germany, IBM Research GmbH in Switzerland and the CentraleSupélec (CS) in France.

In the following sections we will present key information about the major development achieved during the lifetime of the project, and shed light on the next actions needed to bring the technology to a higher maturity level (e.g. exploitable systems for specific markets). The abstract is organized along the following topics:

1. Motivation for developing the PHRESCO technology from market, business and societal challenges perspectives;

2. An overview on the PHRESCO concept and results achieved;

3. The technical barriers that still need to be removed;

4. Conclusions and outlook for future work and cooperation.

\section{Motivation for Developing the PHRESCO Technology}

Millions of networked sensors are being embedded in the physical world in devices such as mobile phones, smart meters, automobiles, industrial machines etc., which sense, create and communicate data. Social media sites, smartphones, and other consumer devices have allowed billions of individuals around the world to contribute to the amount of "Big data" available today. According to some estimates [3], the amount of data produced worldwide is doubling every two years; it is expected to increase from 4.4 zettabytes (or 4.4 trillion gigabytes) in 2013 to 44 zettabytes in 2020. Big data analytics have the potential to identify efficiencies that can be made in a wide range of sectors, and to lead to innovative new products and services, competitiveness and economic growth. Moreover, given the intrinsic temporal nature of involved data, for which the RC approach (as a paradigm for modeling RNNs) is chosen and identified as suitable, ultra-efficient photonic implementation could take great advantage thereof.

There are growing demands from many different market sectors to analyze "Big data" and utilize it to generate value (i.e. health care, transport, insurance, marketing, intelligence agencies, modelling of complex systems, etc.). For instance, modern medicine collects huge amounts of information about patients through imaging technology (scans, MRI), genetic analysis (DNA microarrays), and other forms of diagnostic equipment. By applying data analysis or mining to data sets for large numbers of patients, medical researchers are gaining fundamental insights into the genetic and 
environmental causes of diseases, and creating more effective means of diagnosis. It has been envisioned that the potential annual value to the US health care with the use of "Big Data" is $\$ 300$ billion, two-thirds would be in the form of reducing expenditures by about $8 \%$. Another example is the public sector administration in Europe where the use of "Big Data" has a potential annual value of $\$ 250$ billion, $\$ 150$ billion of which would come from only operational efficiency improvements. In the transport area, navigation companies collect billions of traffic measurement points daily; using this data to reduce congestion could result, by 2020, in worldwide savings of US $\$ 500$ billion in time and fuel, and 380 megatons of $\mathrm{CO}_{2}$ emissions.

The huge potential of Big Data cannot be fully unlocked using traditional computing technologies based on the Von-Neumann architecture. Faster and smarter computing concept will need to be developed to unleash the full potential of Big Data.

Reservoir computing (RC) is one of the concepts that has proven its efficiency to perform tasks where traditional approaches fail. As a simple description for RC, the input data are forced in a so-called reservoir to interact with themselves, and this interaction results in an output pattern that can be more easily classified. Although there is a clear search for new computing paradigms, very few examples show a hardware implementation of it. Most of the time, the physical implementation is performed in CMOS to emulate e.g. neuronal behavior, and is intrinsically limited in power efficiency and speed. RC is one of the rare concepts of efficient hardware realizations of cognitive computing, shown to fit natively into a specific technology, namely integrated photonics.

In 2014, optical RC networks based integrated photonic circuits were realized by the Photonics Research Group at Ghent University who is a partner of PHRESCO. In their ground-breaking work the optical reservoir is a passive network of silicon photonic waveguides, interferometers, and splitters. The PHRESCO projects capitalized on this discovery, and aimed at bringing photonic reservoir computing to the next level of maturity. A new RC chip was co-designed, including innovative electronic and photonic components that will enable major breakthrough in the field. The main building blocks of the project consist of: (i) scale up the optical RC systems with the development of two generations of chip consisting of larger networks (18 and 60 nodes), with an on-chip readout and novel training approaches (ii) build an all-optical chip based on the unique electro-optical properties of new materials (iii) implement new learning algorithms to exploit the capabilities of the RC chip. The hardware integration of beyond state-of-the-art components with novel system and algorithm design will pave the way towards a new era of optical, cognitive systems capable of handling huge amount of data at ultra-low power consumption.

\section{PHRESCO's Concept and Results Achieved}

\subsection{Overview on the PHRESCO Concept}

In order to develop compact and multifunctional photonic networks integrated on silicon substrates, optically active elements such as non-linear elements, weightining elements, amplifiers and detectors were implemented for the first time into the photonic 
reservoir computing (PhRC) that was scaled up to reach 60 nodes (4 times larger than previously fabricated). Therefore, an all-optical PhRC was built to boost the speed of the system while strongly reducing their power consumption. The project overview and concept is illustrated in Fig. 1. Moreover, the system layout and learning algorithms such as training methods were developed and investigated.

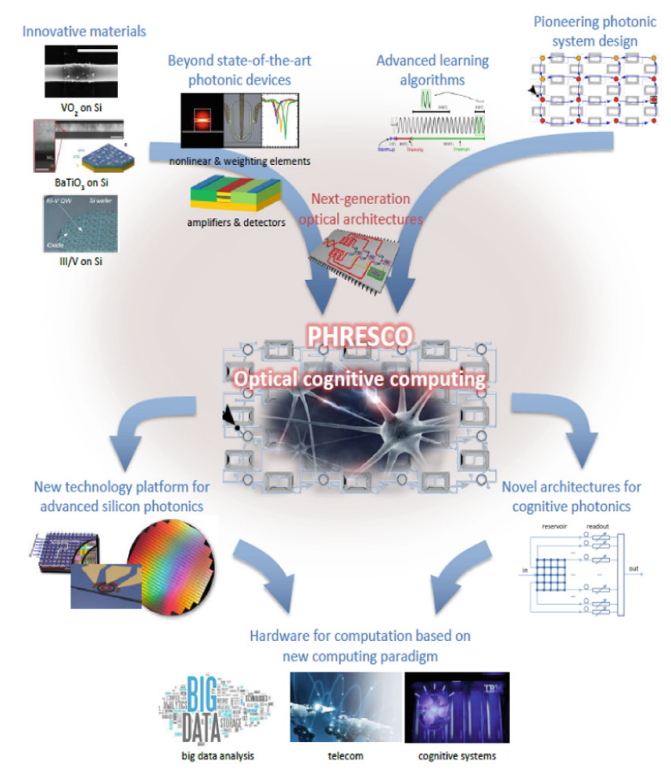

Fig. 1. Overview and concept of the PHRESCO project.

\subsection{Overview on the Achieved Results}

In the first phase of the PHRESCO project, a first generation reservoir with 18 nodes and integrated readout was designed and fabricated. The chip was designed as a $2 \times 9$ photonics swirl reservoir integrated with 3 readout technologies: a silicon readout, a vanadium oxide (VO2) readout and a barium titanate (BaTiO3) readout. The first set of prototype chips were produced and characterised by transmission experiments. The first end-to-end measurements of the reservoir states were attempted. Particle Swarm Optimization training method has been used to simulate the training of a $2 \times 9$ reservoir in order to establish a baseline, as well as a first fall-back training method for our prototypes. For a more efficient and fast training, a method based on the inversion of the output nonlinearity of the reservoir has been developed.

At the level of the individual components, the extension of the current functionalities of the new proposed materials was studied. Regarding the $\mathrm{VO}_{2}$ technology, optical and electro-optical characterization of $\mathrm{VO}_{2}$-Si photonic structures (1st generation of test chips) was done. However, it was not possible to stabilize a non-volatile switching of the $\mathrm{VO}_{2}$ layers. On the other hand, the performance of $\mathrm{BaTiO}_{3}$ based programmable weights was studied. Electro-optical characterization of $\mathrm{BaTiO}_{3}-\mathrm{Si}$ 
devices confirmed the Pockels effect and the reversible and non-volatile switching behavior of the $\mathrm{BaTiO}_{3}$ layers. An experimental platform has been designed and developed for characterization of non-linear behavior on $\mathrm{BaTiO}_{3}$ thin films. The test experiments show that polarized light can be coupled and detected before and after the sample respectively.

During the $2^{\text {nd }}$ phase of the PHRESCO project, the main efforts were focused on the co-integration of different components on the photonic chips, i.e. weighting elements, amplifier and nonlinear elements. Regarding the weighting elements, we established a procedure to erase the history of a weight, independent of the previous settings. In the case of $\mathrm{VO}_{2}$, we have demonstrated a tunable transverse electric pass polarizer based on hybrid $\mathrm{VO}_{2} / \mathrm{Si}$ devices showing the potential of $\mathrm{VO}_{2}$ as an active material for opto-electronic devices, see Fig. 2 [4].
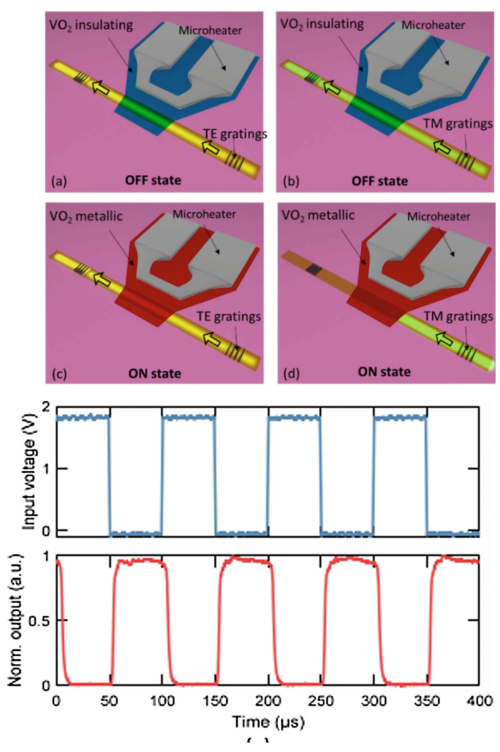

Fig. 2. (Top) concept art of the proposed TE pass polarizer as a function of the input polarization and $\mathrm{VO}_{2}$ state. (Bottom) electrical driving signal applied to the microheater and photo detected signal at the output of the polarizer for TM polarization.

In parallel, large efforts of the consortium was dedicated to the design of the second-generation chip, see Fig. 3. This part of the work included simulations to optimize the performance of components, architecture layout, best input strategy, best way to readout and combine the different states and power budget studies. The available prototype was upscaled to larger networks (60 nodes), with an on-chip readout and novel training approaches, and the scalability and cascadability of these RC networks were investigated. From the analysis of performance of the reservoir it could be concluded that a chaining architecture can be optimal in order to leverage the 
performance of electro-optical and fully optical passive photonic reservoir computing systems. The work is currently ongoing and the experimental results will be presented in a separate paper.

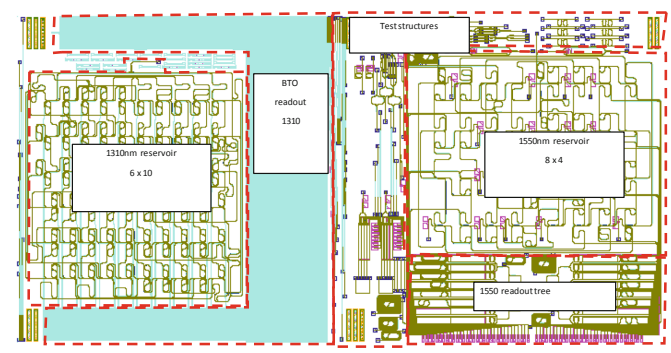

Fig. 3. Low-loss reservoir, $2^{\text {nd }}$ generation mask layout

At the level of the individual components, we greatly progressed in the fabrication of III/V based amplifiers, which showed optical gain at wavelength $1300 \mathrm{~nm}$, and could successfully couple light between Si-photonic structures and III/V devices placed at different photonic layers. Finally, we also examined the non-linearity properties in the BaTiO3 layer by using an experimental setup specifically designed for the study of the photorefractive effect.

\section{The Technical Barriers That Still Need to Be Removed to Move to an Exploitable System}

Although the PHRESCO project has advanced considerably the state of the art of reservoir computing, there are still several technical barriers that need to be removed in order to bring the concept closer to a marketable product. Below the list of the most important barriers that need to be removed in a future work in collaboration with interested stakeholders in the topic: (1) The number of nodes need to be scaled further up (at least >1000 nodes); (2) The footprint of the demonstrator needs to be reduced with novel designs (currently the reservoirs in PHRESCO are already in the range of $1 \times 1 \mathrm{~cm}$ ); (3) Better photonic nonlinear elements need to be design (while keeping losses low). Tackling those barriers with stakeholders who are interested to co-develop the PHRESCO technologies further, will be essential to demonstrate the concept on a system level suitable for specific market applications.

\section{Conclusions and Outlook for Future Work and Cooperation}

To date, PHRESCO have generated a number of innovations that were translated into patents and high impact scientific publications, mainly related to materials, components and the concept/modelling of the demonstrator. Although it's expected that functional 
demonstrators will be obtained by the end of the project at a small prototype level, obviously there will be still a need to develop those demonstrators further before they can be introduced into the market. This abstract gives an overview on the status of the developed technologies so far, and the barriers that still need to be removed. This abstract lays down the foundations for a potential cooperation with stakeholders who could be interested to co-develop the concept further to a system level targeting specific applications. The market applications that are targeted are cloud-based analytics of big and unstructured data, cognitive signal processing, healthcare and financial sector.

\section{References}

1. Verstraeten, D., et al.: An experimental unification of reservoir computing methods. Neural Netw. 20(3), 391-403 (2007). https://doi.org/10.1016/j.neunet.2007.04.003

2. Vandoorne, K., et al.: Experimental demonstration of reservoir computing on a silicon photonics chip. Nat. Commun. 5, 3541 (2014). https://doi.org/10.1038/ncomms4541

3. Turner, V.: The digital universe of opportunities (2014). https://www.emc.com/leadership/ digital-universe/2014iview/index.htm

4. Sanchez, L., et al.: Experimental demonstration of a tunable transverse electric pass polarizer based on hybrid VO2/silicon technology. Opt. Lett. 43(15), 3650-3653 (2018). https://doi. org/10.1364/OL.43.003650 